\title{
DISFUNÇÃO TEMPOROMANDIBULAR E DOR CERVICAL: COMORBIDADES?
}

\author{
TEMPOROMANDIBULAR DYSFUNCTION AND NECK PAIN: COMORBID CONDITIONS?
Alexandre Bittencourt Moreira ${ }^{a^{*}}$, Marcia Morete ${ }^{b^{*}}$, Francisco Cordon $^{\mathrm{c}^{* *}}$, William Rafael Malezan ${ }^{\mathrm{d}^{*}}$ \\ afisioterapeutadaatm@gmail.com, bmarciamorete@gmail.com, cfc.cordon@gmail.com, ddr.rafaelmalezan@hotmail.com \\ *Hospital Albert Einstein - São Paulo (SP), Brasil \\ **Equilíbrio, Instituto Transdisciplinar para o Estudo da Dor - São Paulo (SP), Brasil
}

Data de recebimento do artigo: 31/03/2015

Data de aceite do artigo: 19/11/2015

\section{RESUMO}

Introduçáo: A dor cervical é o sintoma mais comum encontrado nas disfunçóes cervicais e está frequentemente relacionada à manutenção de posturas inadequadas. Os índices de tais disfunçóes vêm aumentando nos últimos anos. É estimado que cerca de \%50 dos adultos experimentarão dor cervical em algum momento da vida. Objetivo: Com todo esse contexto, o presente estudo tem como objetivo buscar publicações na literatura para elucidar a relação entre disfunçáo temporomandibular e dores cervicais como comorbidades. Materiais e métodos: Trata-se de uma revisão integrativa da literatura, relativa à dor cervical e à disfunção temporomandibular como comorbidades. A busca na literatura foi realizada por meio do cruzamento das seguintes palavras-chave: cefaleia; dor cervical; transtornos da articulação temporomandibular; zumbido. Resultados: Encontramos um total de 4804 artigos com descritores individuais. Esses estudos foram, na sua maioria, encontrados na base de dados PubMed (2526 artigos), seguido da base LILACS (652 artigos), BBO (416 artigos) e IBECS (218 artigos). Com uma análise criteriosa, observando os critérios de inclusão e exclusão, chegamos a um total de 12 artigos selecionados. Conclusóes: Mais pesquisas devem ser feitas identificando as comorbidades sistêmicas relacionadas às disfunçóes temporomandibulares e às dores orofaciais para que o tratamento seja holístico e, assim, assertivo em um menor intervalo de tempo.

Palavras-chave: Disfunção temporomandibular; comorbidades; dor.

\section{ABSTRACT}

Introduction: Neck pain is the most common symptom found in cervical disorders and is often related to inadequate postures. The rates of such disorders have increased in recent years. It is estimated that about $50 \%$ of adults will experience neck pain at some point in their life. Objective: Within this context, the present study undertakes a literature search to elucidate if temporomandibular dysfunction and neck pain could be considered as comorbidities. Materials and methods: The research was based on an integrative literature review related to neck pain and temporomandibular disorders as comorbidities. It was carried out using the following keywords: headache; neck pain; temporomandibular joint disorders; tinnitus. Results: We found a total of 4,804 articles with individual descriptors. These studies were taken from PubMed database (2,526 articles), LILACS (652 articles), BBO (416 articles) and IBECS (218 articles). After a meticulous selection, observing the criteria for inclusion and exclusion, we came up with 12 articles for this research. Conclusions: More research should be made toidentify the systemic comorbidities related to temporomandibular disorders and orofacial pain, aiming to develop a holistic and assertive treatment in shorter time.

Keywords: Temporomandibular dysfunction; comorbidities; pain. 


\section{Introdução}

De acordo com a International Association for the Study of Pain (IASP), dor é "uma sensação ou experiência emocional desagradável, associada a um dano tecidual real ou potencial ou descrita em termos de tal dano" ${ }^{1}$.

Podemos observar então que a dor é um mecanismo protetor. Ocorre sempre que qualquer tecido é lesionado, fazendo o indivíduo reagir para remover o estímulo doloroso ${ }^{2}$. Entretanto, pode se manifestar com frequência mesmo na ausência de agressóes teciduais vigentes ${ }^{3}$.

Nesse contexto, encontramos a dor cervical, que é o sintoma mais comum encontrado nas disfunçóes cervicais e está frequentemente relacionada à manutenção de posturas inadequadas. Os índices de tais disfunçōes vêm aumentando nos últimos anos ${ }^{4}$. É estimado que cerca de $50 \%$ dos indivíduos adultos experimentarão dor cervical em algum momento da vida ${ }^{5}$.

Especificamente como causa das dores cervicais, temos a disfunção temporomandibular (DTM), que é muito frequente e acomete o sistema estomatognático e é definida como uma doença ou grupo de doenças com vários subgrupos de etiologia e tratamentos específicos que afetam a articulaçáo temporomandibular (ATM), músculos mastigatórios e estruturas adjacentes; estima-se que aproximadamente $5 \%$ das pessoas acometidas procuram auxílio por causa de suas queixas principais: dor na regiáo pré-auricular, fundo dos olhos, região temporal, ângulo da mandíbula, além de dores na face e cefaleias ${ }^{6,7}$.

É observada uma relação direta no posicionamento da cabeça e a dimensão vertical em repouso, uma vez que o mau posicionamento da cabeça sobre o pescoço cria um desequilíbrio musculoesquelético, e assim a posição de repouso da mandíbula pode ser alterada. Dessa forma, um procedimento que melhore a postura da cabeça reduzirá as dores cervicais e os sintomas das DTMs ${ }^{8}$.

Com todo esse contexto, o presente estudo tem como objetivo buscar publicaçóes na literatura para elucidar a relaçáo entre disfunçáo temporomandibular e dores cervicais como comorbidades.

\section{Metodologia}

Trata-se de uma revisão integrativa da literatura, relativa à dor cervical e à disfunção temporomandibular como comorbidades, realizada segundo as seguintes etapas: elaboração da pergunta norteadora; busca ou amostragem na literatura; coleta de dados; análise crítica dos estudos incluídos; discussão dos resultados; apresentação da revisão integrativa?.

A busca na literatura foi realizada por meio do cruzamento das palavras-chave disponíveis nos descritores em saúde (DECS): cefaleia; dor cervical; transtornos da articulação temporomandibular; zumbido.

Foi realizada a seguinte associação dos descritores: associaçáo: cervicalgia and transtornos da articulação temporomandibular. Foram acessadas as seguintes bases de dados: Bibliografia Brasileira de Odontologia (BBO); Índice Bibliográfico Espanhol de Ciências da Saúde (IBECS); Literatura Latino-Americana e do Caribe em Ciências da Saúde (LILACS) e Publicações Médicas (PUBMED).

A questão norteadora deste trabalho consiste em: "disfunção temporomandibular e dor cervical: comorbidades?”. Foram utilizados os seguintes critérios de inclusão: disponibilidade do artigo na íntegra; publicados em português, inglês e espanhol; publicados no período de 2010 a 2014; realizados com humanos; associação dos descritores por meio de operadores booleanos; títulos referentes aos descritores; leitura classificatória do resumo; leitura do texto na íntegra.

Foram incluídos artigos dos seguintes tipos: revisão narrativa de literatura; revisão sistemática de literatura; pesquisa de campo; relato de experiência e relato de caso, independente da formação profissional do autor. Foram excluídos artigos que não estiveram disponibilizados na íntegra, títulos não condizentes com os descritores e textos sem elemento relevante ou com associação direta a descritores que náo compóem o escopo do trabalho, além de outros tipos de dores ou transtornos senão os dos descritores e alteraçóes de origem patológica ou traumática.

Ressalta-se ainda que houve o cuidado necessário para que os artigos indexados nas bases de dados selecionadas não fossem incluídos duas vezes.

\section{Resultados e discussão}

A representação abaixo elucida a relevância dos temas encontrados (ainda não associados aos descritores e termos de exclusão):

Tabela 1: Total de publicações encontradas nas bases de dados relacionadas com os descritores e sua associação.

\begin{tabular}{cccc}
$\begin{array}{c}\text { Fonte da } \\
\text { publicaçáo }\end{array}$ & Cervicalgia & $\begin{array}{c}\text { Transtornos da } \\
\text { articulaçáo tempo- } \\
\text { romandibular }\end{array}$ & Associaçáo \\
\hline BBO & 10 & 406 & 7 \\
\hline IBECS & 116 & 102 & 1 \\
\hline LILACS & 95 & 557 & 8 \\
\hline PUBMED & 1660 & 1858 & 41 \\
\hline TOTAL & 1881 & 2923 & 57 \\
\hline
\end{tabular}

Após a aplicação de todos os critérios exclusão, obteve-se a seguinte tabela: 
Tabela 2: Total de publicações encontradas nas bases de dados com associação dos descritores e aplicação dos critérios de exclusão.

\begin{tabular}{|ccc|}
\hline $\begin{array}{c}\text { Fonte da } \\
\text { publicaçáo }\end{array}$ & Quantidade & $\%$ \\
\hline BBO & 1 & 8,3 \\
\hline IBECS & 1 & 8,3 \\
\hline LILACS & 1 & 8,3 \\
\hline PubMed & 9 & 75 \\
\hline Total & 12 & 100 \\
\hline
\end{tabular}

Após verificar todos os artigos selecionados, a amostra total foi composta por $12(100 \%)$ publicações, sendo $9(83,3 \%)$ em inglês, $1(8,3 \%)$ em espanhol e 1 $(8,3 \%)$ em português.

A distribuição por ano de publicação foi agrupada segundo os critérios de inclusão, ou seja, entre janeiro de 2010 e dezembro de 2014.

Tabela 3: Total de publicações selecionadas nas bases de dados com associação dos descritores, aplicação dos critérios de exclusão e separadas por ano de publicação.

\begin{tabular}{|ccc|}
\hline Ano & Quantidade & \% \\
\hline 2010 & 3 & 25 \\
\hline 2011 & 4 & 33,3 \\
\hline 2012 & 3 & 25 \\
\hline 2013 & 1 & 8,3 \\
\hline 2014 & 1 & 8,3 \\
\hline Total & 12 & 100 \\
\hline
\end{tabular}

A distribuição dos artigos foi agrupada de acordo com a quantidade publicada por cada periódico.

A partir das buscas realizadas na primeira fase do estudo com pesquisa de bases de dados (BBO, IBECS, LILACS e PubMed), encontramos um total de 4804 artigos com descritores individuais. Esses estudos foram, na sua maioria, encontrados na base de dados PubMed (2526 artigos), seguido da base LILACS (652 artigos), BBO (416 artigos) e IBECS (218 artigos). A partir dessa busca, associamos os artigos aos descritores e, refinando a pesquisa, obtivemos o seguinte achado: PubMed (41 artigos), LILACS (8 artigos), BBO (7 artigos) e IBECS (1 artigo). Com uma análise criteriosa, observando os critérios de inclusão e exclusão, chegamos a um total de 12 artigos selecionados, sendo PubMed (9 artigos), LILACS, BBO e IBECS (1 artigo cada).
Tabela 4: Total de publicações selecionadas nas bases de dados com associação dos descritores, aplicação dos critérios de exclusão e separadas por ano de publicação.

\begin{tabular}{lcc}
\multicolumn{1}{c}{ Periódico } & Quantidade & $\%$ \\
\hline $\begin{array}{l}\text { Jornal da Sociedade Brasileira de } \\
\text { Fonoaudiologia }\end{array}$ & 1 & 8,3 \\
\hline $\begin{array}{l}\text { Nutrición Hospitalaria } \\
\text { Revista da Associação Paulista dos } \\
\text { Cirurgióes Dentistas }\end{array}$ & 1 & 8,3 \\
\hline $\begin{array}{l}\text { Journal of Oral \& Facial Pain and } \\
\text { Headache }\end{array}$ & 1 & 8,3 \\
\hline Dental Clinics of North America & 1 & 8,3 \\
\hline $\begin{array}{l}\text { Compendium of Continuing Education in } \\
\text { Dentistry }\end{array}$ & 1 & 8,3 \\
\hline The Clinical Journal of Pain & 1 & 8,3 \\
\hline Physical Therapy & 1 & 8,3 \\
\hline Manual Therapy & 2 & 16,6 \\
\hline Journal of Oral Rehabilitation & 1 & 8,3 \\
\hline Journal of the American Dental Association & 1 & 8,3 \\
\hline Total & 12 & 100 \\
\hline
\end{tabular}

A disfunção temporomandibular relaciona-se intimamente com a regiáo cervical. Queixas de dor na regiâo da cabeça e pescoço são uma das razóes para os pacientes procurarem o tratamento ${ }^{20}$. Por causa dessa relação complexa e multifatorial, atribui-se abordagens multidisciplinares com uma equipe que inclui médicos de dor, dentistas, acupunturistas, massagista, fisioterapeutas, psicólogos e terapeuta ocupacional ${ }^{19}$.

Anatomicamente, a região cervical é a base e o suporte das estruturas da cabeça e pescoço, e por causa de sua proximidade e inervações comuns, as desordens da coluna cervical podem se manifestar com dores na regiáo orofacial ${ }^{20}$.

Embora haja essa relação direta entre a articulação temporomandibular e a coluna cervical, alteraçóes de postura craniocervical não são observadas em pacientes com ou sem DTM ${ }^{17}$. Uma limitação encontrada é a dificuldade em observar um grupo de indivíduos apenas com DTM sem déficit cervical ${ }^{21}$. Dessa forma, observa-se que a presença de DTM resulta em maior frequência de sintomas dolorosos na região cervical ${ }^{17}$.

Indivíduos com DTM apresentaram uma diminuição da resistência dos músculos extensores cervicais detectados por eletromiografia. Além disso, pacientes com mais dor, mais anos de sintomas e tendo mais deficiência no pescoço e na mandíbula consequentemente apresentam mais fadiga dos músculos extensores cervicais ${ }^{18}$. 
Tabela 5: Estudo sobre a relação de cervicalgia e transtornos da articulação temporomandibular segundo autor, ano, tipo de estudo, proposta de estudo, metodologia, resultado e desfecho.

\begin{tabular}{|c|c|c|c|c|c|}
\hline Autor/ano & $\begin{array}{l}\text { Tipo de } \\
\text { estudo }\end{array}$ & Proposta do estudo & Metodologia & Resultado & Desfecho \\
\hline $\begin{array}{l}\text { Armijo-Olivo et al } \\
2010^{10}\end{array}$ & $\begin{array}{l}\text { Estudo de } \\
\text { campo }\end{array}$ & $\begin{array}{l}\text { Quantitativo para associa- } \\
\text { ção de alteraçôes cervicais e } \\
\text { transtornos da articulação } \\
\text { temporomandibular }\end{array}$ & $\begin{array}{l}\text { Questionário com } 169 \\
\text { mulheres com idade entre } \\
18 \text { e } 50 \text { anos }\end{array}$ & $\begin{array}{c}\text { Alta prevalência de } \\
\text { cervicalgia em pacientes } \\
\text { com DTM }\end{array}$ & $\begin{array}{l}\text { Demostra que pacientes } \\
\text { com DTM apresentam } \\
\text { mais alteraçóes cervicais }\end{array}$ \\
\hline $\begin{array}{l}\text { Armijo-Olivo et al } \\
2010^{11}\end{array}$ & $\begin{array}{l}\text { Estudo de } \\
\text { campo }\end{array}$ & $\begin{array}{l}\text { Quantitativo para associa- } \\
\text { ção de alteraçôes cervicais e } \\
\text { transtornos da articulaçáo } \\
\text { temporomandibular }\end{array}$ & $\begin{array}{l}\text { Questionário com } 172 \\
\text { mulheres com idade entre } \\
18 \text { e } 50 \text { anos }\end{array}$ & $\begin{array}{l}\text { Alta prevalência de } \\
\text { alteraçóes cervicais e } \\
\text { mandibulares em indi- } \\
\text { víduos com DTM }\end{array}$ & $\begin{array}{l}\text { Valida e evidencia } \\
\text { os déficits cervicais } \\
\text { e mandibulares em } \\
\text { pacientes com DTM }\end{array}$ \\
\hline $\begin{array}{l}\text { Svensson et al } \\
2010^{12}\end{array}$ & $\begin{array}{l}\text { Relato de } \\
\quad \text { caso }\end{array}$ & $\begin{array}{l}\text { Determinar a importância e } \\
\text { identificar os mecanismos de } \\
\text { dor na região orofacial }\end{array}$ & $\begin{array}{l}\text { Descrição de caso; mulher } \\
\text { de } 34 \text { anos com escala } \\
\text { de dor } 7 \text { entre } 0 \text { e } 10 \text { na } \\
\text { região da ATM, masseter, } \\
\text { temporais e ângulo da } \\
\text { mandíbula. }\end{array}$ & $\begin{array}{c}\text { Por meio de um } \\
\text { exame clínico apurado, } \\
\text { imagens radiológicas } \\
\text { e histórico completo, } \\
\text { descobriu-se que parte } \\
\text { da dor tratava-se de dor } \\
\text { neuropática pós-cirurgia } \\
\text { da ATM }\end{array}$ & $\begin{array}{l}\text { As dores mais comuns } \\
\text { são nociceptivas e infla- } \\
\text { matórias nessa região, } \\
\text { porém há a possibilidade } \\
\text { de outros tipos de dor } \\
\text { nessa regiāo, o que in- } \\
\text { fluenciará a estratégia de } \\
\text { tratamento e o resultado }\end{array}$ \\
\hline $\begin{array}{l}\text { Armijo-Olivo et al } \\
2011^{13}\end{array}$ & $\begin{array}{l}\text { Estudo de } \\
\text { campo }\end{array}$ & $\begin{array}{l}\text { Determinar se pacientes com } \\
\text { DTM têm aumento da ativi- } \\
\text { dade dos músculos cervicais } \\
\text { superficiais }\end{array}$ & $\begin{array}{l}\text { Questionário, exame clíni- } \\
\text { co e exame de eletromio- } \\
\text { grafia em } 168 \text { indivíduos } \\
\text { com idade entre } 18 \text { e } 50 \\
\text { anos }\end{array}$ & $\begin{array}{l}\text { Há maior atividade } \\
\text { muscular nos múscu- } \\
\text { los ECOM e escaleno } \\
\text { anterior nos indivíduos } \\
\text { com DTM }\end{array}$ & $\begin{array}{l}\text { O aumento da atividade } \\
\text { dos músculos cervicais } \\
\text { pode ser associado com } \\
\text { os distúrbios da cervical } \\
\text { encontrados em pacientes } \\
\text { com DTM }\end{array}$ \\
\hline $\begin{array}{l}\text { Armijo-Olivo et al } \\
2011^{14}\end{array}$ & $\begin{array}{l}\text { Estudo de } \\
\text { campo }\end{array}$ & $\begin{array}{l}\text { Relevância clínica comparada } \\
\text { com a significância estatística }\end{array}$ & $\begin{array}{l}\text { Questionário, exame clíni- } \\
\text { co e exame de eletromio- } \\
\text { grafia em } 154 \text { indivíduos }\end{array}$ & $\begin{array}{l}\text { O método irá revelar } \\
\text { a relevância clínica e a } \\
\text { significância estatística in- } \\
\text { dividualmente, associadas } \\
\text { ou nenhuma das duas }\end{array}$ & $\begin{array}{l}\text { A relevância clínica } \\
\text { deve ser considerada } \\
\text { e comparada com os } \\
\text { resultados obtidos pelas } \\
\text { pesquisas }\end{array}$ \\
\hline $\begin{array}{l}\text { Céspedes et al } \\
2011^{15}\end{array}$ & $\begin{array}{l}\text { Estudo de } \\
\text { campo }\end{array}$ & $\begin{array}{l}\text { Quantificar e relacionar os } \\
\text { sintomas de DTM, limitaçốes } \\
\text { funcionais e autopercepção da } \\
\text { saúde oral }\end{array}$ & $\begin{array}{l}\text { Questionário com } 94 \text { in- } \\
\text { divíduos com idade acima } \\
\text { de } 65 \text { anos }\end{array}$ & $\begin{array}{c}\text { Sintomas inespecíficos } \\
\text { com mais prevalência } \\
\text { foram cervicalgia e } \\
\text { nervosismo }\end{array}$ & $\begin{array}{c}\text { Clara associação dos sinto- } \\
\text { mas de DTM, limitaçôes } \\
\text { funcionais e autopercepção } \\
\text { da saúde oral }\end{array}$ \\
\hline $\begin{array}{l}\text { Micelli } \\
\text { et al } \\
2011^{16}\end{array}$ & $\begin{array}{l}\text { Estudo de } \\
\text { campo }\end{array}$ & $\begin{array}{l}\text { Avaliar as estruturas cervicais } \\
\text { durante o exame } \\
\text { para o diagnóstico das DTMs }\end{array}$ & $\begin{array}{l}\text { Questionário e exame } \\
\text { físico. } 200 \text { pacientes } \\
\text { aleatórios }\end{array}$ & $\begin{array}{c}\text { Alta prevalência de } \\
\text { alterações cervicais em } \\
\text { pacientes com DTM }\end{array}$ & $\begin{array}{l}\text { Cervical deve ser ava- } \\
\text { liada nos pacientes com } \\
\text { diagnóstico de DTM }\end{array}$ \\
\hline $\begin{array}{l}\text { Weber } \\
\text { et al } \\
2012^{17}\end{array}$ & $\begin{array}{l}\text { Estudo de } \\
\text { campo }\end{array}$ & $\begin{array}{l}\text { Investigar a frequência de } \\
\text { sinais e sintomas de alteraçóes } \\
\text { cervicais em indivíduos com e } \\
\text { sem DTM e avaliar a influên- } \\
\text { cia da postura craniocervical } \\
\text { nas DTMs }\end{array}$ & $\begin{array}{c}\text { Questionário com } 71 \\
\text { mulheres com idade entre } \\
19 \text { e } 35 \text { anos }\end{array}$ & $\begin{array}{c}\text { Os indivíduos sem DTM } \\
\text { apresentaram mais altera- } \\
\text { çoes leves na cervical; os } \\
\text { indivíduos com menor } \\
\text { DTM apresentaram } \\
\text { frequência de alteraçōes } \\
\text { moderadas ou graves na } \\
\text { cervical }\end{array}$ & $\begin{array}{l}\text { Não houve diferença na } \\
\text { postura craniocervical } \\
\text { em indivíduos com e } \\
\text { sem DTM. A DTM } \\
\text { resultou em maior fre- } \\
\text { quência de dor cervical }\end{array}$ \\
\hline $\begin{array}{l}\text { Armijo-Olivo et al } \\
2012^{18}\end{array}$ & $\begin{array}{l}\text { Estudo de } \\
\text { campo }\end{array}$ & $\begin{array}{l}\text { Determinar se pacientes com } \\
\text { DTM apresentam fadiga mus- } \\
\text { cular dos músculos extensores } \\
\text { da cervical }\end{array}$ & $\begin{array}{l}\text { Questionário e exame de } \\
\text { eletromiografia em } 151 \\
\text { mulheres com idade entre } \\
18 \text { e } 50 \text { anos }\end{array}$ & $\begin{array}{l}\text { Os tratamentos envol- } \\
\text { vendo a resistência dos } \\
\text { músculos cervicais teriam } \\
\text { um impacto positivo na } \\
\text { funçáo e na dor de pacien- } \\
\text { tes com DTM }\end{array}$ & $\begin{array}{l}\text { Pacientes com DTM } \\
\text { apresentaram menos re- } \\
\text { sistência nos músculos } \\
\text { extensores da cervical }\end{array}$ \\
\hline $\begin{array}{l}\text { Mehta NR } \\
2012^{19}\end{array}$ & $\begin{array}{l}\text { Artigo de } \\
\text { revista }\end{array}$ & $\begin{array}{c}\text { Expor a importância do } \\
\text { tratamento multidisciplinar } \\
\text { nas DTMs }\end{array}$ & $\begin{array}{l}\text { Descrição de atuaçáa em } \\
\text { centro de dor craniofacial }\end{array}$ & $\begin{array}{l}\text { Com uma equipe mul- } \\
\text { tidisciplinar, há maior } \\
\text { eficiência no manejo das } \\
\text { dores craniofaciais }\end{array}$ & $\begin{array}{l}\text { As dores craniofaciais } \\
\text { nunca acometem o } \\
\text { indivíduo de forma } \\
\text { isolada, sendo preciso } \\
\text { uma equipe com várias } \\
\text { especialidades médicas } \\
\text { e terapias de suporte }\end{array}$ \\
\hline $\begin{array}{l}\text { Kumar } \\
\text { et al } \\
2013^{20}\end{array}$ & $\begin{array}{l}\text { Artigo de } \\
\text { revista }\end{array}$ & $\begin{array}{l}\text { Sugere a importância do } \\
\text { diagnóstico correto nas dores } \\
\text { orofaciais }\end{array}$ & Revisão bibliográfica & $\begin{array}{l}\text { Sinais e sintomas po- } \\
\text { dem não ser específicos } \\
\text { para determinado pro- } \\
\text { blema ou distúrbio }\end{array}$ & $\begin{array}{l}\text { Necessidade do diagnós- } \\
\text { tico diferencial a ampla } \\
\text { e complexa etiologia das } \\
\text { dores orofaciais }\end{array}$ \\
\hline $\begin{array}{l}\text { Silveira } \\
\text { et al } \\
2014^{21}\end{array}$ & $\begin{array}{l}\text { Estudo de } \\
\text { campo }\end{array}$ & $\begin{array}{l}\text { Comparar a sensibilidade } \\
\text { dos músculos mastigatórios e } \\
\text { cervicais e a sensibilidade do- } \\
\text { lorosa em área remota (máo) } \\
\text { em pacientes com DTM }\end{array}$ & $\begin{array}{l}\text { Questionário e exame } \\
\text { clínico com } 40 \text { mulheres } \\
\text { com idade entre } 18 \text { e } 50 \\
\text { anos }\end{array}$ & $\begin{array}{l}\text { Aumento da sensibili- } \\
\text { dade na área remota es- } \\
\text { colhida nos indivíduos } \\
\text { com DTM e alteraçóes } \\
\text { cervicais associadas }\end{array}$ & $\begin{array}{l}\text { A importância da } \\
\text { atençăa à cervical e } \\
\text { outras partes do corpo } \\
\text { e não apenas à regiáo } \\
\text { craniofacial }\end{array}$ \\
\hline
\end{tabular}




\section{Considerações finais}

Embora o foco da pesquisa tenha sido a relação de comorbidades entre as disfunçóes temporomandibulares e dor cervical, observamos que as disfunçôes da coluna cervical apresentam-se como uma importante comorbidade, sendo a dor cervical e a fadiga muscular os principais sinais e sintomas dessa disfunção.

Essa hiperatividade da musculatura cervical nos indivíduos com DTM é um importante achado da eletromiografia, uma vez que a estratégia para o sucesso do tratamento das disfunçōes temporomandibulares deve englobar uma abordagem multidisciplinar com ação direta na região cervical.

Mais pesquisas devem ser feitas identificando as comorbidades sistêmicas relacionadas às disfunções temporomandibulares e às dores orofaciais para que o tratamento seja holístico e, assim, assertivo em um menor intervalo de tempo.

\section{Referências}

1. International Association for the Study of Pain. Disponível em: http://www.iasp-pain.org/Taxonomy\#Pain.

2. Guyton AC, Hall JE. Tratado de fisiologia médica. $11^{\mathrm{a}}$ ed. Rio de Janeiro: Elsevier; 2006.

3. Kay TM, Gros A, Goldsmith CH, Rutherfars S, Voth S, Hoving JL, et al. Exercises for mechanical neck disorders. Cochrane Database Syst Rev 2012; 8: CD004250.

4. Soares JC, Weber P, Trevisan ME, Trevisan CM, Rossi AG. Correlação entre postura da cabeça, intensidade da dor e índice de incapacidade cervical em mulheres com queixa de dor cervical. Braz J Phys Ther 2012; 19(1):68-72.

5. Hogg-Johnson S, Van Der Velde G, Carroll LJ, Holm LW, Cassidy JD, Guzman J, et al. The burden and determinants of neck pain in the general population. J Manipulative Physiol Ther 2009; 32(2): S46-60. doi:10.1016/j.jmpt.2008.11.010

6. Portinho CP, Razera MV, Splitt BI, Gorgen ARH, Faller GJ, Collares MVM, et al. Apresentação clínica inicial em pacientes com disfunção temporomandibular. Braz J Craniomaxillo Surg 2012; 15(3): 109-12.

7. Siqueira JTT. As dores orofaciais na prática hospitalar - experiência brasileira. Prática Hospitalar 2006; VIII(48): 85-9.

8. Darling DW, Krauss S, Clasheen-Wray MB. Relationship of head posture and the rest position of the mandible. J Prosthet Dent 1994; 52(1): 111-5.

9. Souza MT, Silva MD, Carvalho R. Revisão integrativa: o que é e como fazer. Revista Einstein 2010; 8(1): 102-6.
10. Armijo-Olivo S, Fuentes J, Costa BR, Major PW, Warren S, Thie NMR, et al. Reduced endurance of the cervical flexor muscles in patients with concurrent temporomandibular disorders and neck disability. Man Ther 2010; 15(6): 58692. doi:10.1016/j.math.2010.07.001

11. Armijo-Olivo S, Fuentes J, Major PW, Warren S, Thie NMR, Magee DJ. The association between neck disability and jaw disability. J Oral Rehabil 2010, 37(9), 670-9. doi:10.1111/j.1365-2842.2010.02098.x

12. Svensson P, Baad-hansen L. The mechanisms of joint and muscle pain. J American Dental Association 2010; 141(6): 672-4. doi:10.14219/jada.archive.2010.0256

13. Armijo-Olivo S, Silvestre R, Fuentes J, Costa BR, Gadotti IC, Warren S, et al. Electromyographic activity of the cervical flexor muscles in patients with temporomandibular disorders while performing the craniocervical flexion test: a cross-sectional study. Phys Ther 2011 Aug; 91(8): 1184-97. doi:10.2522/ptj.20100233

14. Armijo-Olivo S, Warren S, Fuentes J, Magee DJ. Clinical relevance vs. statistical significance: using neck outcomes in patients with temporomandibular disorders as an example. Man Ther 2011; 16(6): 563-72. doi:10.1016/j.math.2011.05.006

15. Almagro Céspedes I, Castro Sánchez AM, Matarán Peñarocha GA, Quesada Rubio JM, Guisado Barrilao R, Moreno Lorenzo C. Disfunción temporomandibular, discapacidad y salud oral en una población geriátrica semi-institucionalizada. Nutr Hosp 2011; 26: 1045-51. doi:10.3305/nh.2011.26.5.4932

16. Micelli ALP, Silva ABW, Silva FA, Oliveira M, Silva LLB, Fernandes JPS, et al. Prevalência de disfuncóes crânio cervicais em pacientes portadores de disfunçóes temporomandibulares. Rev Assoc Paul Cir Dent 2011 Set/Aug; 65(5): 368-74.

17. Weber P, Corrêa ECR, Ferreira FS, Soares JC, Bolzan GP, Silva AMT. Frequência de sinais e sintomas de disfunção cervical em indivíduos com disfunção temporomandibular. J Soc Bras Fonoaudiol 2012; 1(2): 134-9.

18. Armijo-Olivo S, Silvestre R, Fuentes JP, Costa BR, Major PW, Warren $S$, et al. Patients with temporomandibular disorders have increased fatigability of the cervical extensor muscles. Clin J Pain 2012; 28(1): 55-64. doi:10.1097/AJP.0b013e31822019f2

19. Mehta NR. Managing craniofacial pain the multi-disciplinary way. Compend Contin Educ Dent. 2012 Mar; 33(3): 224.

20. Kumar A, Brennan MT. Differential diagnosis of orofacial pain and temporomandibular disorder. Dent Clin North Amer 2013; 57(3): 419-28. doi:10.1016/j.cden.2013.04.003

21. Silveira A, Armijo-Olivo S, Gadotti IC, Magee D. Masticatory and cervical muscle tenderness and pain sensitivity in a remote area in subjects with a temporomandibular disorder and neck disability. J Oral Facial Pain Headache 2014; 28(2): 138-46. doi:10.11607/ofph.1112.

\section{Como citar este artigo:}

Moreira AB, Morete M, Cordon F, Malezan WR. Disfunção temporomandibular e dor cervical. Rev. Aten. Saúde. 2016;14(48):94-98. 